\title{
Um Panorama da Literatura Relacionada às Representações Sociais Publicada em Periódicos Científicos
}

\author{
João Wachelke ${ }^{1}$ \\ Fabíola Rodrigues Matos \\ Gustavo Cerchi Soares Ferreira \\ Rafaela Rannelle de Lima Costa \\ Universidade Federal de Uberlândia, Uberlândia, $M G$, Brasil
}

\begin{abstract}
Resumo
O presente estudo visa a caracterizar parte da produção acadêmica sobre representações sociais publicada em periódicos científicos, de modo a fornecer informações sobre o desenvolvimento e estado atual do campo. Em agosto e setembro de 2013 foram obtidos 2526 resumos de artigos publicados em periódicos indexados em quatro bases de dados, todos sobre representações sociais. Para cada resumo foram registrados o país da instituição do primeiro autor, ano de publicação, disciplina do periódico e informações categorizadas relativas aos grupos e objetos sociais estudados. Os resultados confirmaram e expandiram meta-análises realizadas anteriormente, evidenciando uma contraposição entre estudos aplicados na área de saúde com grupos e objetos relacionados, mais típicos da América do Sul e Brasil e pesquisas psicossociais ligadas à área da psicologia e realizadas por pesquisadores principalmente do continente europeu. Nota-se também um aumento de importância considerável na participação de primeiros autores brasileiros e sul-americanos em relação ao total, tornando-se líder no volume de produção acadêmica na área, substituindo autores provenientes de instituições europeias.
\end{abstract}

Palavras-chave: Representações sociais, produção científica, pesquisa bibliográfica, Bibliometria.

\section{An Overview of the Literature Related to Social Representations Published in Scientific Journals}

\begin{abstract}
The present study aims at characterizing part of the academic production on social representations published in scientific journals, so as to provide information about the development and current state of the field. In August and September 20132526 abstracts of papers published in journals indexed in four databases were retrieved, all of which were about social representations. For each abstract registers were made concerning the country in which the first author's institution was located, publication year, journal discipline and categorized information relative to the studied group and social objects. The results confirmed and expanded previously conducted meta-analyses, giving evidence of a contrast between applied studies in the health area involving related groups and objects, more typical from South America
\end{abstract}

Endereço para correspondência: Instituto de Psicologia, Universidade Federal de Uberlândia, Campus Umuarama, Bloco 2C, Sala 19, Av. Pará 1720, Bairro Umuarama, Uberlândia, MG, Brasil 38400-902. Fone: +55.34.3218-2822. E-mail: wachelke@yahoo.com

Apoio financeiro: Conselho Nacional de Pesquisa e Desenvolvimento Tecnológico (CNPq), Fundação de Apoio à Pesquisa de Minas Gerais (FAPEMIG) e Pró-Reitoria de Pesquisa e Pós-Graduação da Universidade Federal de Uberlândia (PROPP-UFU). 
and Brazil, and psychosocial research linked to the psychology field and carried out mostly by European researchers. A considerable increase in the importance of the participation of Brazilian and South American authors relative to the total production was also noticed, replacing authors from European institutions as leaders according to that criterion.

Keywords: Social representations, scientific production, bibliographic research, Bibliometrics.

\section{Una Visión General de la Literatura Relacionada con las Representaciones Sociales Publicadas en Revistas Científicas}

\section{Resumen}

El presente estudio tiene como objetivo caracterizar parte de la producción académica sobre las representaciones sociales publicada en revistas científicas, para proporcionar información sobre el desarrollo y el estado actual del campo. En agosto y septiembre de 2013, fueron recuperados 2526 resúmenes de artículos publicados en revistas indexadas en cuatro bases de datos, todos sobre las representaciones sociales. Para cada uno se hicieron registros sobre el país de la institución del primer autor, año de publicación, la disciplina de la revista y los grupos y objetos estudiados. Los resultados confirmaron y ampliaron meta-análisis realizados antes, e muestran un contraste entre estudios aplicados en el área de la salud con grupo y objetos afines, más típicos de América del Sur y Brasil, e investigaciones psicosociales del campo de la psicología llevados a cabo por investigadores europeos. Se observó también un aumento considerable en la importancia de la participación de autores de Brasil y de América del Sur en relación con la producción total, en sustitución de autores de instituciones europeas como líderes de acuerdo a ese criterio.

Palabras clave: Representaciones sociales, producción científica, investigación bibliográfica, Bibliometría.

Este artigo apresenta um panorama de parte da produção acadêmica mundial relacionada às representações sociais publicada em periódicos científicos até meados de 2013. As representações sociais são teorias do senso comum sobre assuntos do cotidiano que são compartilhadas e elaboradas por grupos (Jodelet, 1989; Moscovici, 1984). A expressão representação social geralmente associa-se à teoria das representações sociais, um paradigma de pesquisa da psicologia social que se originou da tese de doutorado de Serge Moscovici (1976) sobre as crenças compartilhadas da população francesa sobre a psicanálise nos anos 50 .

Várias décadas após a proposição da teoria, um estudo das características dos artigos que empregaram as representações sociais poderia fornecer uma avaliação do desenvolvimento internacional da teoria e da sua popularização por diversas disciplinas com o passar dos anos. Além das qualidades científicas de uma teoria isto é, os testes que ela enfrenta continuamente para provar-se um modelo plausível para compreender a realidade social-, sua difusão entre disciplinas, países e continentes poderia dar evidências de seu valor como um modelo para abordar problemas científicos e esclarecer acerca das necessidades das comunidades acadêmicas de diferentes contextos.

Dentre as diversas escolas sociopsicológicas, a teoria das representações sociais é comumente considerada uma forma sociológica de psicologia social (Farr, 1998; Moscovici, 1989). Sua especificidade reside na atenção dada a processos históricos e de comunicação na construção e transmissão de conhecimento do dia-a-dia (Jodelet, 1989; Rouquette, 1998), o que a diferencia de variedades sociopsicológicas do mainstream, mais focadas em processos cognitivos (De Rosa, 1993; Duveen \& De Rosa, 1992).

Uma variedade de escolas e perspectivas de representações sociais pode ser encontrada, enfatizando aspectos diferentes do fenômeno, de abordagens etnográficas para entender a relação 
entre a apropriação individual de uma cultura grupal até estudos experimentais do gerenciamento individual das informações que são compartilhadas por membros do grupo (para trabalhos ilustrando a diversidade metodológica da pesquisa em representações sociais, ver Abric, 2003; Moreira, Camargo, Jesuíno, \& Nóbrega, 2005).

Trata-se de uma perspectiva que é frequentemente aberta ao diálogo interdisciplinar. Muito da pesquisa é dedicado a temas transdisciplinares aplicados como saúde e educação (Jodelet, 2011). Além disso, o conceito de representação social é um esforço para ultrapassar as fronteiras sociopsicológicas dos fenômenos para atingir níveis mais sociais de explicação, atraindo a atenção de acadêmicos das outras ciências sociais. Deve ser lembrado também que representação é um conceito popular nesses campos independentemente de seu uso no contexto do paradigma de Moscovici; este foi na verdade inspirado pela noção de Durkheim (1898) de representação coletiva. Assim, a despeito de diferenças importantes, uma sobreposição considerável é esperada à medida que as várias noções de representação dos modelos das ciências sociais preenchem papeis semelhantes nos sistemas teóricos.

Originalmente uma teoria psicológica social da França, a teoria das representações sociais foi bem recebida por alguns pesquisadores de países europeus como Portugal, Itália e Inglaterra. Autores que classificam as variedades de psicologia social que são propostas ao redor do mundo associam a teoria à psicologia social europeia, devido à ênfase em processos ideológicos e intergrupais (Farr, 1993; Jesuíno, 2000). Posteriormente ela espalhou-se por outros continentes, encontrando sucesso particular em países latino-americanos e no Canadá francófono. Sua popularização foi muito mais modesta nos Estados Unidos, possivelmente devido à preferência por uma psicologia social cognitiva individualista e experimental, a qual contrasta com o estudo comparativo de representações sociais históricas, compartilhadas e culturalmente elaboradas (Farr, 1996).

A apreciação do estado da arte da pesquisa em representações sociais pode fornecer infor- mações acerca dos contextos de difusão do estudo de fenômenos relacionados e dos aspectos que são privilegiados. Há alguns exemplos bem conhecidos de tais esforços na literatura. Um primeiro projeto nessa direção foi possivelmente a bibliografia geral de representações sociais organizada por Jodelet e Ohana (1989), publicada num livro clássico do campo. A bibliografia foi apenas uma lista de recursos alinhados com a teoria das representações sociais até aquele momento.

Algumas meta-análises conhecidas da literatura sobre representações sociais foram fornecidas por Annamaria de Rosa. Em 1992, ela revisou a literatura crescente em psicologia do desenvolvimento alinhada com representações sociais e cognição social e discutiu seus pontos de contato e divergência, incluindo uma categorização dos objetos sociais estudados ou perspectivas temáticas levadas em consideração pelas pesquisas (De Rosa, 1992).

De Rosa e D'Ambrosio (2008) conduziram uma meta-análise de 1731 resumos apresentados em inglês, francês e espanhol nas primeiras oito Conferências Internacionais sobre Representações Sociais, as principais conferências bienais relacionadas à teoria das representações sociais. Além de codificar informações bibliográficas clássicas como a conferência em que cada trabalho foi apresentado e a nacionalidade do primeiro autor, elas categorizaram cada resumo em termos das áreas temáticas privilegiadas pela investigação ou o tipo de tratamento dado à teoria das representações sociais. No que diz respeito à última categoria, as autoras avaliaram se somente um tratamento geral foi dado ao fenômeno das representações sociais, ou se paradigmas específicos ligados à teoria foram enfocados - tais como estrutura, gênese, ou metateoria. Os resultados indicaram um contraste entre trabalhos de autoria de europeus, que focaram proporcionalmente mais em aspectos teóricos e referências relacionadas à teoria paradigmática das representações sociais, e trabalhos escritos por autores latino-americanos, que tinham natureza aplicada e empírica e lidavam com as representações sociais de modo genérico.

No mesmo espírito, Madiot (2013) apresentou uma análise dos resumos em língua francesa 
das 11 Conferências Internacionais bienais sobre representações sociais. Seu corpus consistiu de 2549 resumos. Sua análise também enfocou as escolas teóricas que foram identificadas nas comunicações e escolhas metodológicas. Quanto aos objetos de representação social, os mais frequentes foram trabalho, educação e saúde. Em termos de grupos, as populações de crianças, pais, professores, cuidadores e profissionais de saúde foram privilegiadas.

Mais recentemente, De Rosa (2013) apresentou um panorama da análise metateórica de um corpo extensivo de literatura sobre a teoria das representações sociais. Em janeiro de 2012, o banco de dados consistia de 6938 referências, 2700 das quais eram artigos e capítulos de livros que passaram por meta-análise detalhada. Ela apresentou os resultados de uma análise metateórica baseada em 2065 fontes bibliográficas, incluindo 956 capítulos de livros, 936 artigos de periódicos, 65 trabalhos apresentados em conferências, 57 capítulos de teses de doutorado, 46 relatórios ou manuscritos universitários e 5 documentos da web, todos publicados de 1952 a 2009. Seus resultados apontam para uma tendência de publicar contribuições teóricas em capítulos de livros e pesquisas empíricas em artigos de periódicos, e também confirmam o interesse interdisciplinar ao redor da teoria das representações sociais. Dentre os principais assuntos das contribuições publicadas, a análise identificou questões teóricas e metodológicas, a relação da teoria das representações sociais com a psicologia social e outras disciplinas, saúde-doença, comunicação, e relações interpessoais e intergrupais. A pesquisa preocupa-se com a avaliação de aspectos específicos da teoria das representações sociais que são enfatizados pelos trabalhos. O projeto diferencia entre estudos que incorporam os conceitos teóricos da teoria das representações sociais e outros que referem-se ao fenômeno de maneira genérica, possivelmente desconectados da teoria.

Houve também algumas análises da literatura em representações sociais providenciadas por acadêmicos brasileiros. Os primeiros estudos de representações sociais no Brasil foram publicados de 1982 a 1997 (Sá \& Arruda, 2000). Ar- ruda (1987) publicou uma primeira revisão dos trabalhos pioneiros relacionados à teoria ou ao fenômeno das representações sociais no nordeste do Brasil no período anterior às JIRS, as Jornadas Internacionais sobre Representações Sociais, eventos realizados também de dois em dois anos na América Latina. Sá e Arruda (2000) analisaram 867 trabalhos científicos englobando artigos, teses, dissertações e resumos publicados entre 1988 e 1997; eles concluíram que o volume da produção estava crescendo rápido à época, com a expansão da publicação de livros. À medida que as JIRS foram se consolidando, algumas revisões enfatizaram a avaliação metodológica dos resumos publicados. Essas análises trouxeram à luz a natureza aplicada da produção brasileira, com atenção especial a questões de saúde e educação (Arruda, 2005; Camargo, Wachelke, \& Aguiar, 2007; Jodelet, 2011). O interesse interdisciplinar no estudo das representações sociais também é evidente a partir da existência de revisões de dissertações e teses de pós-graduação alinhadas com a teoria nos campos da enfermagem (A. M. F. Silva, Martini, \& Becker, 2011; S. E. D. Silva, Camargo, \& Padilha, 2011) e educação (Sousa, 2002).

Pode-se perceber então que os acadêmicos da teoria das representações sociais estão monitorando constantemente a literatura alinhada com seu paradigma para mapear diferenças e transformações nas práticas científicas a respeito da teoria. Contudo, representação social é uma expressão que pode levar a pesquisas e teorias relevantes fora do modelo proposto por Moscovici, em campos básicos e aplicados. O estudo das representações sociais em um modo não ligado diretamente à teoria das representações sociais não é um problema, já que antropólogos, sociólogos e outros estudiosos podem investigar construções simbólicas legitimamente, bem como suas consequências e determinações, com outras diretrizes. Propomos um panorama da pesquisa sobre o construto das representações sociais em todas as ciências sociais, independente de divergências teóricas. Adicionalmente, focalizaremos nossa atenção numa fonte bibliográfica específica: periódicos revisados por pares. Essa escolha justifica-se pela avaliação criteriosa por que cada 
manuscrito passa no processo gerenciado por um editor e geralmente dois pareceristas, pertencendo ao conselho editorial ou tendo status ad hoc. Os artigos são aceitos somente depois que sua relevância e correção tenham sido asseguradas por meio de controle de qualidade, em um processo que é geralmente mais rigoroso - ou pelo menos mais uniforme, compartilhando padrões - que o que é observado às vezes com livros ou anais de conferências. Propomos que há espaço para uma análise temática de artigos de periódicos sobre representações sociais num conjunto mais amplo de fontes internacionais que as que foram observadas até então.

Além das variáveis clássicas como a localização das instituições dos primeiros autores ou a disciplina de filiação dos periódicos, será dada ênfase à identificação das populações e grupos levados em consideração em estudos de representações sociais. Cada estudo de representações sociais se refere necessariamente a um grupo que representa e a um objeto, um tópico da vida social com alguma saliência para o grupo, que é representado (Jodelet, 1989). Há uma variedade significativa de populações e objetos estudados por estudos de representações sociais.

Uma análise dos grupos e objetos que inspiram mais estudos e a associação dessas escolhas com contextos institucionais e geográficos específicos podem trazer luz às práticas de pesquisa relacionadas às representações sociais. Análises semelhantes foram realizadas, geralmente por meio de uma categorização de áreas temáticas amplas (Camargo et al., 2007; De Rosa, 2013; De Rosa \& D'Ambrosio, 2008). De Rosa (2013) indicou que cada área temática, tal como saúde-doença ou economia-trabalho-organização, inclui vários subtópicos. Os últimos podem geralmente ser traduzidos como objetos ou categorias de objetos. Em contaste, Madiot (2013) apresentou uma distribuição de frequência diretamente no nível de classes de objetos sociais, mas não verificou quaisquer associações com outras variáveis, restringindo um pouco sua investigação.

O objetivo da presente contribuição é realizar uma análise temática exploratória dos resumos de artigos de periódicos relacionados ao estudo de representações sociais. Artigos de bases de dados internacionais relativas às ciências psicológicas, sociais e da saúde foram incluídas, e as variáveis consideradas dizem respeito ao contexto geográfico do primeiro autor, às disciplinas ligadas aos periódicos, aos períodos de publicação e aos grupos e objetos que foram estudados.

\section{Método}

\section{Fontes de Dados}

Um estudo documental foi realizado. Recuperamos todos os resumos em inglês de quatro grandes bases de dados de resumos que resultaram de uma busca com os termos "representação social" ou "representações sociais" nos campos de título, resumo e palavras-chave. Ainda que a escolha de bases de dados tenha se baseado em alguma diversidade de disciplinas e regiões e levado em conta recursos importantes, deve ficar claro que o esforço não reflete o universo completo das bases de dados científicas. As bases de dados escolhidas foram PsycINFO (American Psychological Association); Scientific Eletronic Library Online (Scielo. org); MEDLINE Complete (EBSCOHost); e Sociological Abstracts (ProQuest). As escolhas justificam-se pela cobertura de disciplinas importantes e áreas geográficas, incluindo uma base de dados por campo disciplinas principal que é comumente associado ao estudo das representações sociais (psicologia, ciências sociais, saúde) e também uma base de dados geral incluindo periódicos latino-americanos de todos os campos, fornecendo sobreposição significativa com bases de dados específicas de disciplinas mas ao mesmo tempo acrescentando alguns periódicos mais recentes ou mais regionais. PsycINFO é a principal base de dados da psicologia e também inclui periódicos de outras ciências humanas e sociais, Scielo é uma base de dados generalista que cobre majoritariamente periódicos sul-americanos - mas também inclui a África do Sul e países ibéricos -, Sociological Abstracts cobre as ciências sociais e MEDLINE contém pesquisas relacionadas ao campo da saúde. 


\section{Procedimento}

A busca e codificação de resumos ocorreram de agosto a setembro de 2013. Os conteúdos dos resumos foram verificados, de modo que somente aqueles relacionados a representação social no sentido de conhecimento compartilhado foram mantidos para análise. Resumos relacionados a outros significados da expressão representação social, por exemplo participação política ou representatividade popular, foram excluídos. Além disso, já que o conteúdo das bases de dados selecionadas se sobrepõe, as repetições também foram excluídas. Um total de 3359 resumos foram identificados primeiramente, e 2526 deles foram casos únicos relacionados ao significado referido das representações sociais. Esses 2526 resumos formaram a amostra retida para análise. Os coautores realizaram a busca e recuperação de artigos independentemente e o primeiro autor verificou e discutiu os resultados para chegar a um consenso em termos de quais unidades de pesquisa deveriam ou não ser incluídas.

As informações relacionadas a cada resumo foram codificadas num banco de dados para análise posterior. As seguintes informações foram registradas: ano de publicação, disciplina do periódico em que o resumo foi publicado, país do primeiro autor, objeto social estudado e grupo social estudado.

As informações relativas aos grupos e objetos foram categorizadas de acordo com seu conteúdo, agrupando temas semelhantes em categorias mais amplas. No total, 23 categorias foram formuladas para os objetos e 16 para os grupos. Para testar os sistemas de categorias, dois codificadores, ambos autores do presente manuscrito, categorizaram 191 resumos $(7,5 \%$ do total) independentemente tanto para grupos quanto objetos. A proporção de concordância intercodificador foi de $79 \%$ para objetos e $88,5 \%$ para grupos. Ajustes foram feitos ao sistema para resolver categorizações ambíguas. Depois, um dos codificadores procedeu a categorizar os grupos e o outro foi responsável pela categorização dos objetos. O primeiro autor foi responsável por verificar a categorização e discutir casos ambíguos para decidir suas categorias apropriadas.
As vinte e três categorias relativas a objetos sociais (exemplos entre parênteses) foram: cidadania e direitos humanos (Direitos Humanos, cidadania); competências e habilidades (habilidades do professor, inteligência); cultura e etnia (cultura grega, budismo); desenvolvimento humano (adolescência, concepção humana); drogas (consumo de tabaco, cocaína); educação (ambiente escolar, cursos de pós-graduação); emoções (medo, felicidade); eventos históricos (colonização europeia, crise econômica de 2008); grupo racial (pessoas negras, preconceito racial); meio ambiente (água, meio ambiente); controvérsias e eventos do cotidiano (acidentes de trabalho; urbanismo); posição social (pessoas sem-teto, minorias); trabalho (condições de trabalho, autonomia profissional); saúde e bem- estar (AIDS, qualidade de vida); questões econômicas (pobreza, comércio); questões políticas (democracia, ação coletiva); relações sociais (amizade, vida conjugal); sexualidade e gênero (corpo, opções sexuais); tecnologia (internet, tecnologias reprodutivas); território e comunidade (grupos ligados a viver ou nascer em regiões específicas); violência e crime (agressão, assédio); outros (objetos com frequências baixas não se encaixando nas outras categorias); ausente (resumos em que os objetos não se aplicavam - por exemplo em um ensaio teórico geral - ou não foram informados).

As dezesseis categorias de grupos foram: clientes ou consumidores; comparações culturais (estudos com grupos de pessoas de nacionalidades diferentes); estudantes (estudantes universitários e escolares); grupos étnicos; membros familiares; grupos associados a posições sociais (classe alta, baixa); grupos associados a preferências, gostos ou hábitos (por exemplo, fumantes, praticantes de esportes); grupos etários; residentes de regiões ou grupos ligados a território (por exemplo habitantes de uma vizinhança específica); multicategoria (consideração de duas ou mais pertenças a categorias ao mesmo tempo); população geral (sem especificação além do sexo dos membros do grupo); pacientes e portadores de doenças crônicas; profissionais com formação educacional específica (por exemplo, profissionais de educação e saúde); trabalhado- 
res e empregados em geral; outros (grupos com baixas frequências não se encaixando noutras categorias); ausente (resumos em que os grupos não se aplicavam - por exemplo em pesquisas documentais - ou não foram informados).

\section{Resultados}

\section{Período de Publicação e Localização do Primeiro Autor}

No que diz respeito à relação entre período de publicação e o continente de filiação do primeiro autores, a variável ano de publicação foi recodificada como década de publicação, resultando em cinco modalidades: anos 60 e 70 (respondendo conjuntamente por apenas 13 resumos), anos 80, 90, 2000 e 2010-meados de 2013. Nos anos 60 e 70, 12 dos 13 resumos tiveram o primeiro autor filiado a uma universidade europeia. No decorrer dos anos $80(n=92)$ e $90(n=454)$ a predominância de autores "europeus" continuou, com 84,8\% e $66,3 \%$, mas mostrando uma emergência clara de autores situados na América do Norte $(8,7 \%$ nos anos 80 e $10,3 \%$ nos 90 ) e um crescimento mais importante na América do Sul $(5,4 \%$ nos 80 e $18 \%$ nos 90$)$.

Dos anos 2000 em diante, resumos chefiados por pesquisadores de instituições sul-americanos assumem a ponta. Nos anos $2000(n=$ 1285), os primeiros autores da América do Sul correspondiam a $45,4 \%$ do total, enquanto sua proporção é de mais da metade $(55,8 \%)$ dos 682 resumos publicados no período de 2010 a 2013. A diminuição da proporção de estudos chefiados por pesquisadores baseados na Europa continua (39,7\% nos anos 2000, 32,4\% em 2010-2013). A proporção de resumos com autores da América do Norte é estável (9,2\% nos anos 2000, 9,5\% em 2010-2013).

No nível dos países, primeiros autores do Reino Unido publicaram a maior proporção de estudos nos anos 80 (25\%), seguidos por autores da França (20,7\%) e Itália (13). Os primeiros autores do Brasil foram responsáveis por apenas $3,2 \%$ dos estudos publicados dessa década. $\mathrm{O}$ padrão inverteu-se gradativamente. Nos anos 90, os primeiros autores da França foram responsá- veis por $16,3 \%$ dos resumos, seguidos pelo Reino Unido (15,6\%) e Brasil $(13,2 \%)$. Nos anos 90, primeiros autores de instituições brasileiras tomaram a frente com $34,6 \%$ dos estudos publicados, enquanto os primeiros autores da França e Reino Unido só corresponderam a 12,4\% e 7,1\% dos resumos. No período de 2010-2013, a diferença em proporção cresceu mais: os primeiros autores do Brasil publicaram 39,4\% dos estudos, enquanto que os franceses foram responsáveis por $10,7 \%$. Os autores da Argentina publicaram $6,4 \%$ dos resumos no período, e os britânicos fizeram $6,1 \%$.

Em todos os outros continentes, a proporção nunca ultrapassa $2,4 \%$ em nenhum período estudado, e atualmente eles são menos de um por cento no período mais recente. Para autores da Oceania, não houve resumos publicados nos anos 60 e 70 nem nos 80 . As proporções para os anos 90 foram de $2,4 \%, 19,9 \%$ para os 2000 e $0,8 \%$ para $2010-2013$. Os resultados relativos à Ásia foram semelhantes: nada até os anos 80 , e então $1,5 \%$ para os $90,2,4 \%$ nos 2000 e $0,4 \%$ em 2010-2013. Quanto aos primeiros autores ligados a instituições africanas, os primeiros resumos foram publicados nos anos 90 (1,3\% e então houve $0,9 \%$ nos 2000 e $0,4 \%$ em 2010 2013). Finalmente, quatro resumos chefiados por um autor da América Central foram publicados nos anos 2000, três em 2010-2013 e um nos anos 80 .

\section{Localização do Primeiro Autor e Disciplina do Periódico}

A maior proporção de pesquisas relacionados às representações sociais foi publicada em periódicos de psicologia em todos os continentes. Em termos dos resumos que tinham primeiros autores europeus $(n=1123), 66,7 \%$ dos estudos foram publicados em periódicos desse campo, seguidos pelos periódicos de ciências sociais $(8,7 \%)$ e saúde $(5,6 \%)$. A clara predominância da psicologia também foi observada em contribuições de autores de continentes com um número menor de resumos: Oceania $(n=42)$ teve $73,8 \%$, enquanto a Ásia $(n=41)$ teve $70,7 \%$.

A distribuição dos estudos com autores principais norte e sul-americanos não foi tão contras- 
tante. Quanto aos resumos sul-americanos ( $n=$ $1052), 30,7 \%$ dos estudos foram publicados em periódicos de psicologia, 18,4\% foram recuperados de periódicos interdisciplinares de saúde, 17,6\% apareceram em periódicos de enfermagem, $8,2 \%$ em publicações de ciências sociais e $7,1 \%$ em periódicos de educação. Já dos resumos norte-americanos $(n=239), 23,8 \%$ foram de periódicos de psicologia, $18,4 \%$ de periódicos de saúde, $15,4 \%$ das ciências sociais, $10,4 \%$ de periódicos específicos da sociologia e $6,6 \%$ foram publicados em periódicos de educação.

As distribuições das disciplinas relativas aos resumos com primeiros autores africanos e centro-americanos foram mais pulverizadas. Quanto aos resumos africanos $(n=21), 8$ foram de periódicos de psicologia, 4 de saúde, 4 das ciências sociais e 2 da medicina. Finalmente, 2 dos 8 resumos centro-americanos foram de psicologia e dois de periódicos de medicina.

No nível dos países, as duas localizações predominantes dos primeiros autores foram $\mathrm{o}$ Brasil $(n=776)$ e a França $(n=334)$. A distribuição de resumos dos autores situados na França mostram uma predominância nítida do espaço dos periódicos de psicologia (69,4\%), seguidos pelos de sociologia $(7,7 \%)$, ciências sociais em geral $(6,5 \%)$ e saúde $(5 \%)$. No Brasil, a distribuição é mais homogênea entre as áreas principais. Os periódicos de psicologia lideram com $32 \%$, enquanto enfermagem segue com $23 \%$, saúde tem $20,6 \%$ e educação $6,5 \%$. Os periódicos de psicologia têm uma predominância clara nos resumos de primeiros autores britânicos ( $n$ total $=220$; proporção de artigos em periódicos de psicologia: 64,6\%), bem como nos estudos de autores italianos ( $n=124$, psicologia: 79,8\%), espanhóis $(n=92$, psicologia: $63 \%)$ e portugueses $(n=84$, psicologia: 71,4\%). Os países seguintes em frequência tem um perfil diferente. As maiores proporções de resumos de primeiros autores localizados na Colômbia $(n=84)$ foram de periódicos de saúde $(28,5 \%)$, e o mesmo foi observado para autores mexicanos $(n=86$, saúde: $23,2 \%)$.

\section{Localização do Primeiro Autor, Disciplina do Periódico e Proporções de Objetos e Grupos Estudados}

Deve-se notar que dos 2526 resumos recuperados, $848(33,6 \%)$ não apresentaram informação acerca de um grupo social específico, seja devido a se tratarem de ensaios gerais ou à ausência dessa informação. As análises relativas aos grupos sociais estudados lidaram apenas com os casos com categorias de grupos identificáveis ( $n=1678$ ou $66,4 \%$ do total). Quanto aos objetos sociais, 515 resumos $(20,4 \%)$ não apresentaram informações identificáveis, significando que os resumos analisados compreenderam um total de 2011 , ou $79,6 \%$ do total. Isso quer dizer que nas análises seguintes, $100 \%$ em termos dos grupos sociais estudados somam 1678 resumos, e 100\% dos grupos sociais representa 2011.

A Tabela 1 apresenta dois tipos separados de informação: a distribuição percentual dos objetos e grupos sociais estudados nos resumos, organizados de acordo com a localização da instituição do primeiro autor. Somente os continentes, categorias de objetos e grupos com frequências maiores são mostrados. Relativo a cada continente ou país apresentado, uma coluna de proporção cumulativa indica quantos dos resumos são observados na tabela. Uma linha final (Total) apresenta a distribuição na amostra global de resumos.

Acerca dos objetos, o padrão mais notável envolve a predominância clara de estudos lidando com questões de saúde. Trabalho é o segundo assunto mais saliente, mas está presente somente em pouco mais de seis por cento dos resumos. Para os três continentes na tabela, saúde e bem-estar é a categoria privilegiada, com um perfil mais pronunciado em resumos de autores localizados na América do Sul. No que tange aos autores situados em países específicos, os do Brasil apresentam um perfil diferenciado em termos de uma preferência por objetos de saúde e bem-estar. Os resumos de autores colombianos, britânicos, mexicanos, portugueses e argentinos também se concentram ao redor de saúde e bem-estar, mas alguns temas salientes podem ser observados nesses países: violência e crime 


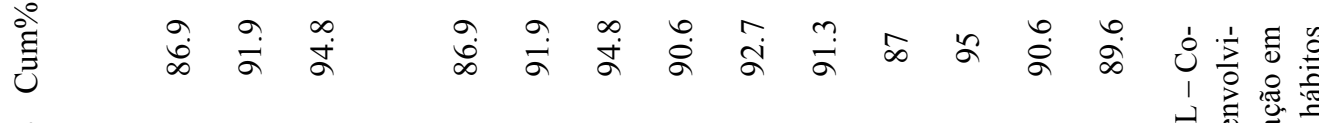

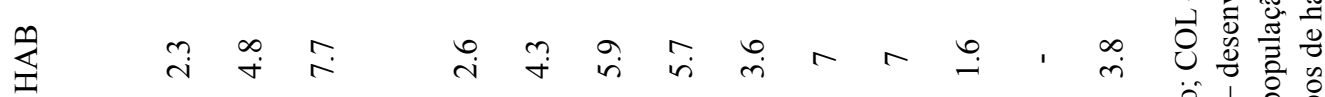

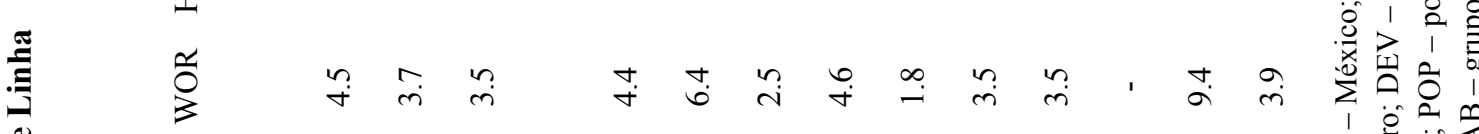

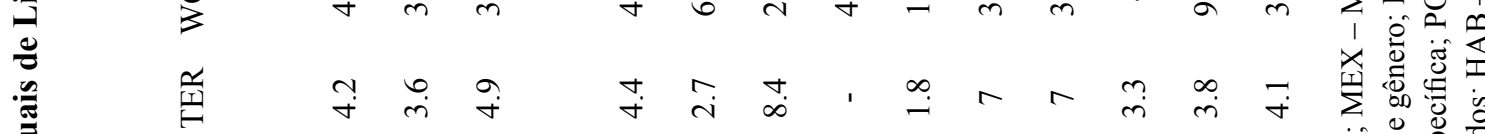


(Reino Unido e Colômbia), sexualidade e gênero (México, Portugal), trabalho (Argentina). Em contraste, os países restantes têm distribuições de resumos mais variadas e equilibradas em termos de categorias de objetos.

A predominância de estudos de saúde na América do Sul e Brasil também se reflete nos grupos sociais investigados. Pode ser observado que estudos de autores localizados no Brasil dão preferência ao estudo de grupos de profissionais com formações específicas, como médicos, professores e enfermeiras, seguidos por estudantes de todos os tipos e pacientes médicos. Nos estudos europeus e norte-americanos, estudos com populações de estudantes prevalecem, possivelmente ligados a questões de educação ou testagem básica de teorias, já que essas populações são mais acessíveis. Resumos e autores britânicos, espanhóis e colombianos têm altas proporções de estudos de população em geral também.

A Tabela 2 apresenta os perfis dos objetos e grupos sociais estudados por disciplina do periódico. Olhando simultaneamente os dois lados da tabela, três perfis emergem claramente. Resumos publicados em periódicos de saúde (incluindo periódicos interdisciplinares de saúde, medicina e enfermagem) privilegiam o estudo de grupos de profissionais e/ou pacientes, e enfocam nitidamente em objetos relacionados a saúde e bem-estar. Resumos de outras ciências humanas e sociais (psicologia, sociologia, ciências sociais em geral) também têm importante proporção de estudos focando questões ligadas a saúde, mas tendem a estudar grupos de estudantes (psicologia e ciências sociais) ou a população geral (sociologia). Resumos publicados em periódicos de educação lidam principalmente com objetos relacionados à educação e amostras de estudantes e professores. Resumos de periódicos interdisciplinares gerais possuem um perfil mais diversificado tanto em termos de grupos quanto de objetos.

Para ter um entendimento compreensivo das principais dimensões de associação, uma análise de correspondências múltiplas foi realizada. A análise de correspondências múltiplas é uma técnica descritiva que sintetiza uma matriz indivíduos (linhas) $\times$ variáveis (colunas) numa decomposição de um número menor de fatores; cada fator descreve oposições em termos de perfis diferentes de indivíduos ou variáveis. $\mathrm{O}$ primeiro fator é o mais importante, explicando as maiores porções da inércia (o termo empregado para referir-se à variância) seguido pelo segundo, terceiro, e assim por diante. Os fatores podem ser representados geometricamente em mapas em que pontos específicos (perfis individuais ou de variáveis) podem ser projetados, tornando possível capturar a estrutura dos dados (Greenacre, 2007; Husson, Lê, \& Pagès, 2011). No presente caso, os indivíduos estatísticos, ou casos, eram os resumos, e as variáveis ativas eram as categorias de grupos e objetos. Para ajudar com a interpretação dos eixos, algumas variáveis suplementares relativas aos resumos (que não participaram no cálculo da análise) foram projetadas no mapa: o continente em que a instituição do primeiro autor estava localizada, a década de publicação e a disciplina do periódico. A análise foi realizada com o programa R (R Core Team, 2013) e o pacote GDAtools (Robette, 2014). Somente categorias de grupos e objetos com frequências maiores que $1 \%$ foram incluídas como ativas nas análises. Além disso, categorias "outros" também foram configuradas como suplementares, devido à dificuldade para interpretá-las.

Analisaremos o primeiro plano fatorial, que inclui os dois fatores mais importantes. $\mathrm{O}$ primeiro fator teve um autovalor de 0,71 , dando conta de $4,68 \%$ da inércia total. O segundo fator, com um autovalor de 0,66 , representou $4,36 \%$ da inércia. Os dois fatores juntos explicaram $9,03 \%$ da inércia. Devido ao grande número de dimensões incluído na análise de correspondências múltiplas, as taxas de inércia dos eixos são normalmente baixas e não refletem bem sua importância. Jean-Paul Benzécri propôs um modo alternativo de calcular a inércia para apreciar melhor a importância dos primeiros eixos, priorizando autovalores maiores que a média (Le Roux \& Rouanet, 2010, pp. 39-40). De acordo com o cálculo modificado, o primeiro fator explica $28,6 \%$ da inércia e o segundo é responsável por $16,6 \%$; ambos juntos explicam $45,2 \%$ da inércia total. 


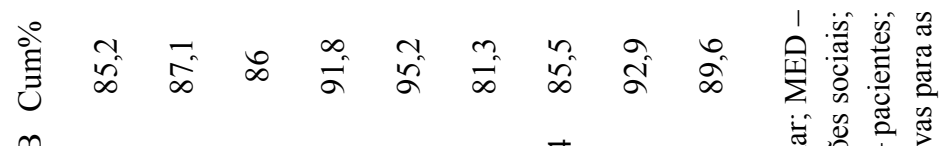

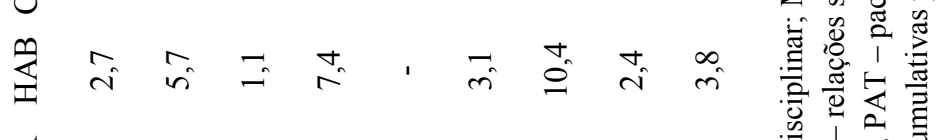

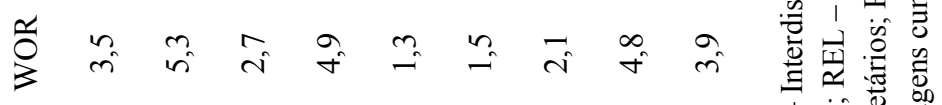

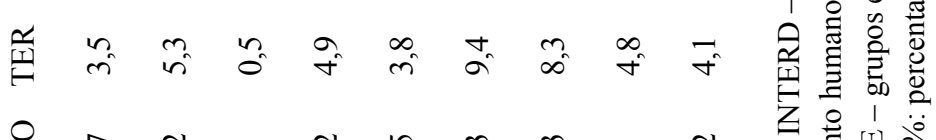

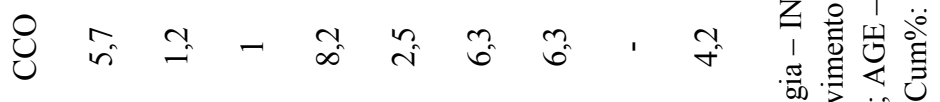

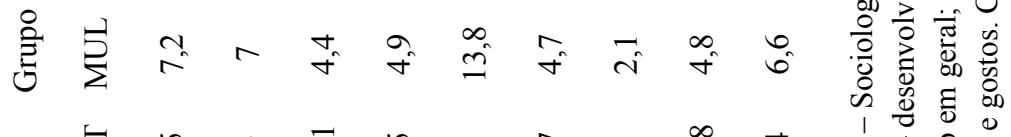

$$
\begin{aligned}
& \text { 灾 }
\end{aligned}
$$

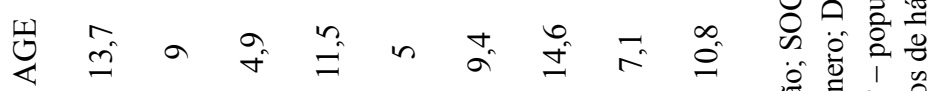

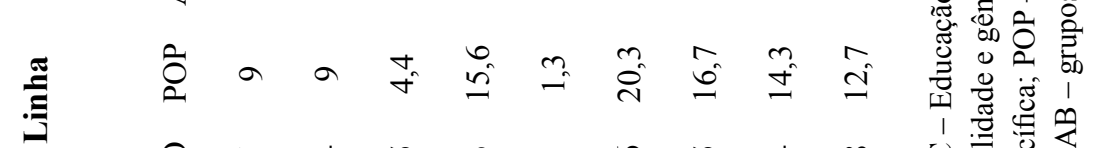

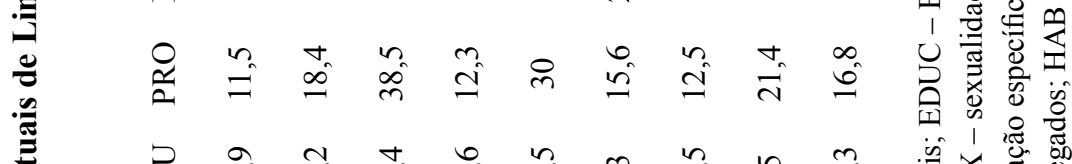

$$
\begin{aligned}
& \text { हैं }
\end{aligned}
$$

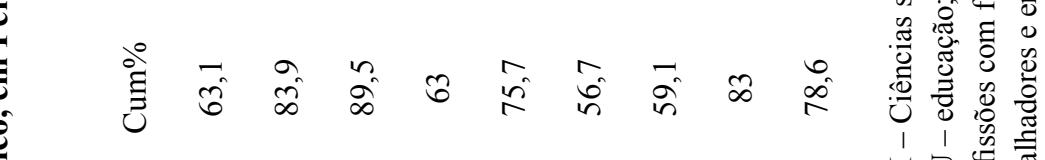

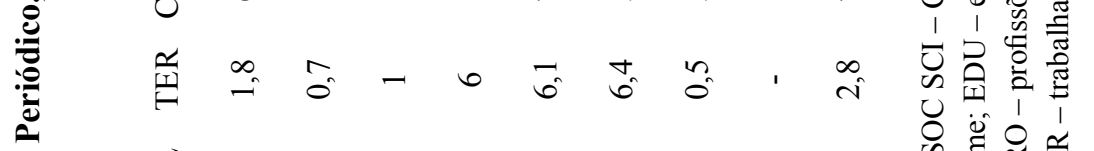

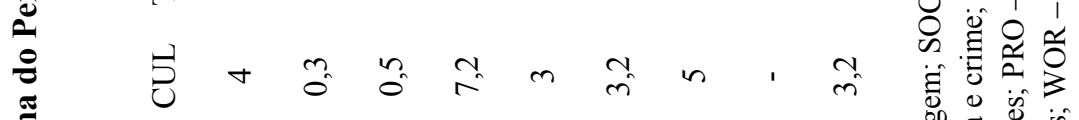

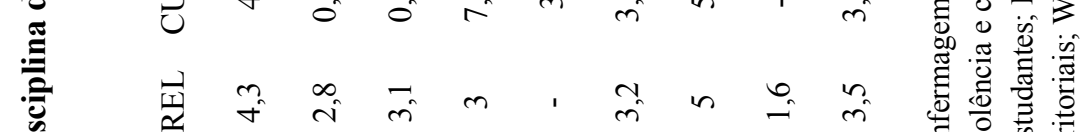

$$
\begin{aligned}
& \text { قू }
\end{aligned}
$$

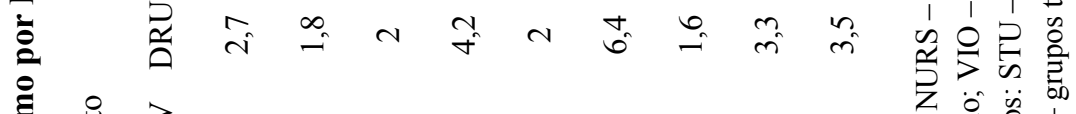

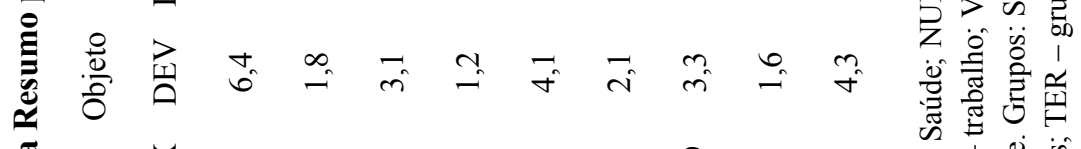

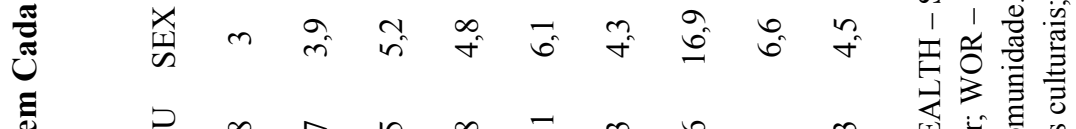

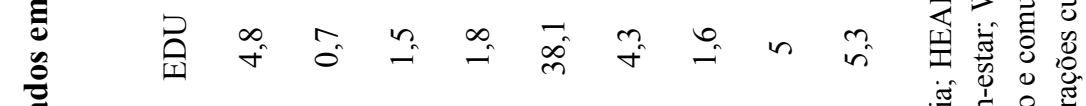

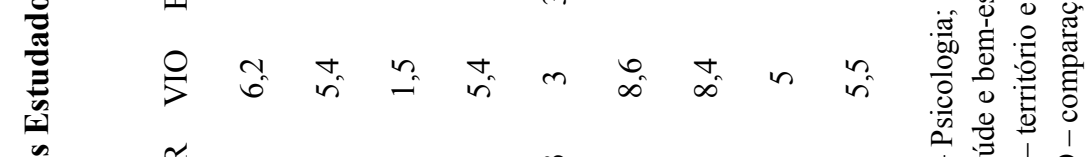

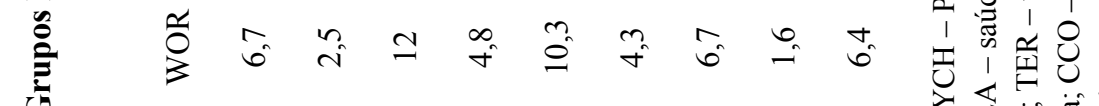

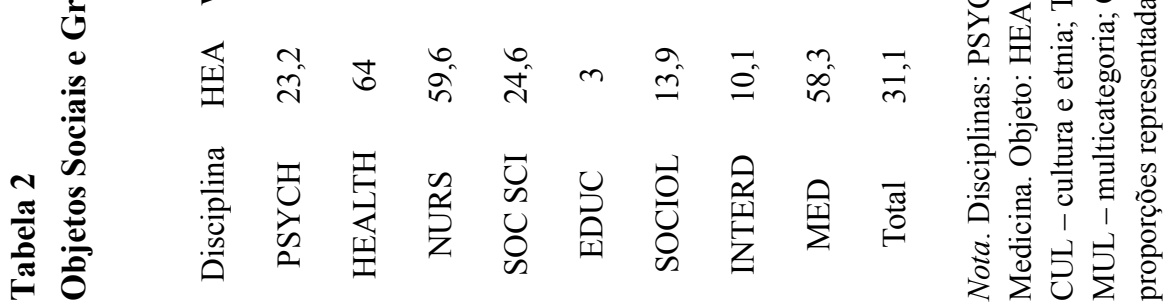


A Figura 1 mostra o mapa relativo aos dois primeiros fatores da análise de correspondências múltiplas. As categorias ativas que são mostradas têm contribuições significativas ao fator que são maiores que a média (100/33 categorias ativas $=3,03$ ). As categorias suplementares mostradas todas tem valores maiores que 3,29 ou menores que $-3,29$, o que indica um ponto de corte sig- nificativo restritivo $(p=0,001)$ no teste-de-valores apresentado por Lebart, Morineau e Piron (1997). Na Figura 1, um sinal de sublinhar (“_”) próximo a um rótulo de categoria indica que a contribuição fatorial para o fator 1 é maior que a média, assim como uma barra vertical ("|") sinaliza uma contribuição importante para o fator 2 . Um sinal de positivo (“+”) indica contribuições maiores que a média em ambos os fatores.

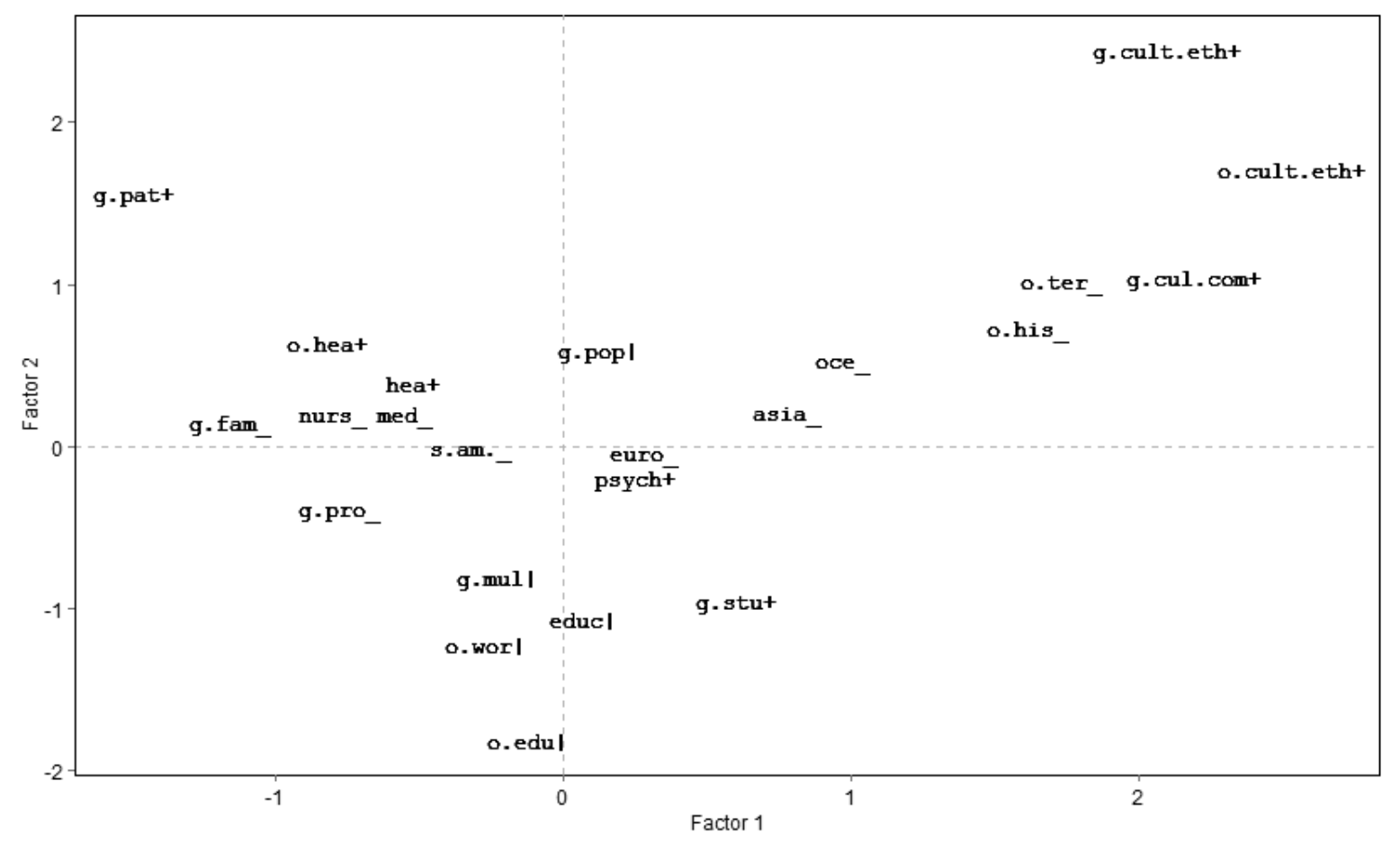

Figura 1. Análise das correspondências múltiplas baseada numa matriz dos resumos $\times$ categorias de grupos (g.) e objetos (o.).

Nota. Continentes: s.am. - América do Sul; euro - Europa; oce - Oceania; asia - Ásia. Objetos: o.hea - saúde e bem-estar; o.wor - trabalho; o.edu - educação; o.cult.eth - cultura e etnia; o.ter - território e comunidade; o.his - eventos históricos. Grupos: g.stu - estudantes; g.pro - profissões com formação específica; g.pop - população geral; g.pat - pacientes; g.mul multicategoria; g.cult.com - comparações culturais; g.fam - membros da família; g.cult.eth - grupos étnicos. Disciplinas de periódicos: nurs - Enfermagem; med - Medicina; hea - Saúde; psych - Psicologia; educ - Educação. Contribuição significativa para os fatores: _: Fator 1 (eixo horizontal); | : Fator 2 (eixo vertical); + : Fatores 1 e 2.

A distribuição dos pontos na nuvem dos indivíduos (não mostrada) é muito semelhante à dos pontos relativos às categorias ativas na Figura 1. Revela um padrão que é chamado efeito ferradura, que pode ser interpretado como uma dominância do primeiro fator, isto é, a maior parte da informação relevante em termos de oposições está apresentada no primeiro fator. O segundo fator então opõe perfis extremos do primeiro fator a outros perfis intermediários, e os fatores seguintes continuam refinando a interpre- tação do primeiro fator mas refletem o mesmo fenômeno (Lebart et al., 1997). A segunda dimensão, contudo, tem alguma relevância já que complementa o contraste geral do primeiro fator (Greenacre, 2006).

Pode ser observado que o principal contraste em termos de perfis de pesquisa presente nos resumos é o da pesquisa sociopsicológica e social básica, situada nas coordenadas positivas do fator 1 , e pesquisa aplicada relacionada à saúde no lado negativo. Os objetos de interesse por parte 
dos pesquisadores sociais envolvem uma proporção maior que a média de assuntos de território e comunidade, bem como tópicos históricos, culturais e étnicos. Os grupos privilegiados em termos de comparação com o perfil da amostra geral são grupos pertencendo a diferentes realidades culturais, para objetos culturais, históricos e territoriais, e estudantes de vários níveis. As pesquisas com essas características foram associadas mais fortemente à instituição do primeiro autor na Europa, Ásia e Oceania, e corresponderam a mais publicações em psicologia. Por outro lado, o polo aplicado enfocou em objetos relacionados à saúde e grupos de profissionais (médicos, enfermeiros), membros de famílias e pacientes. Uma maior proporção de primeiros autores alinhados com essas preocupações é de instituições sul-americanas. Os veículos de publicação nesse caso foram periódicos relacionados à saúde.

O segundo fator opõe pesquisas publicadas sobre objetos de saúde e educação com grupos de estudantes e filiações multicategoriais a estudos sobre representações sociais da saúde com grupos de pacientes, e de modo menos pronunciado com estudos sobre cultura ou etnia com amostras da população geral e grupos ligados a cultura e etnia. A região do eixo ligada à educação envolve uma associação com publicação em periódicos de educação, e menos fortemente com periódicos de psicologia, enquanto a região superior associa-se mais à publicação em periódicos de saúde. $\mathrm{O}$ segundo fator pode ser interpretado como a oposição entre pesquisa aplicada em educação, com interesse específico em realidades profissionais e de formação, e um conjunto incluindo pesquisa aplicada em saúde e algumas pesquisas relacionadas a cultura, associadas a uma definição mais aberta de grupos e à relação de pacientes com saúde e doença.

\section{Discussão}

Os resultados da análise de conteúdo dos resumos de artigos de periódicos publicados sobre representações sociais até 2013 tornam possível identificar uma predominância de primeiros autores localizados nos continentes europeu e sul- -americano. Historicamente, o crescimento na proporção de autores das instituições sul-americanas é notável. No nível nacional, o Brasil é atualmente o líder em termos da localização do primeiro autor, seguido pela França.

Nesse ponto, alguém poderia chegar à conclusão que os sul-americanos, e especialmente os brasileiros, estão "conquistando" o navio das representações sociais. Porém, deve ser percebido que os resultados são restritos às bases de dados incluídas no estudo. Enquanto buscamos considerar bases de dados generalistas bem como algumas principais relacionadas aos campos da saúde, social e psicológico, ao mesmo tempo o SciELO é uma base de dados baseada na América Latina que poderia contribuir para sobrerrepresentar publicações dessa região a partir dos anos 90 - ela foi criada em 1997 - na nossa análise (Packer et al., 1998). Mas plausivelmente essa sobrerrepresentação pode ser uma correção de uma sub-representação prévia, dado que os periódicos latino-americanos tinham número e visibilidade reduzidos. O fato é que atualmente o número de periódicos dessa região é muito maior que em décadas anteriores, bem como sua representação nas principais bases de dados internacionais (Alonso-Gamboa \& Russell, 2012; Collazo-Reyes, 2014).

De todo modo, é seguro concluir que a produção sul-americana (e brasileira) sobre representações sociais publicada em periódicos científicos é muito importante. Talvez não seja mais o caso de se referir tão firmemente ao campo das representações sociais como uma forma "europeia" de psicologia social, ou pelo menos somente europeia. Nossos dados fazem parecer mais que as representações sociais poderiam estar conseguindo cidadania brasileira por "naturalização".

Porém, um olhar mais próximo dos objetos e grupos estudados revela que a pesquisa conduzida em ambos os contextos é muito diferente. Há uma associação maior que a média entre primeiros autores europeus e o estudo de questões culturais, étnicas, históricas e territoriais com grupos de estudantes e membros de culturas ou nações diferentes. Pesquisas desse tipo tendem a ser mais presentes em periódicos 
de psicologia. As pesquisas de autores ligados à Oceania e Ásia vão na mesma direção, com uma variedade de assuntos e grupos de interesse mas com mais ênfase que o perfil geral em termos de pesquisas ligadas a assuntos societais. Em contraste, as pesquisas lideradas por autores sediados na América do Sul são claramente aplicadas a questões de saúde. Pode ser observado nos resultados que saúde é um objeto muito importante para todos os contextos investigados, mas ela o é de modo mais pronunciado nos contextos brasileiro e sul-americano. Esse tipo de pesquisa é majoritariamente direcionado ao estudo das representações de profissionais de saúde e pacientes de objetos relacionados à saúde e geralmente publicados em periódicos relacionados a saúde.

As particularidades das pesquisas realizadas em contextos geográficos já foram identificadas em estudos anteriores (Arruda, 2005; Camargo et al., 2007; De Rosa, 2013; Jodelet, 2011). Essas diferenças poderiam influenciar o desenvolvimento da teoria ou a forma que ela tomará. Jodelet (2011) avaliou a produção brasileira em representações sociais e resistiu a identificar avanços teóricos e metodológicos radicais associados a ela; por outro lado, ela entende que os pesquisadores brasileiros combinam as várias contribuições de autores europeus enquanto que ao mesmo tempo fornecem uma integração criativa das abordagens clássicas para esclarecer problemas práticos que são relevantes para contextos regionais. Jodelet acredita que o mesmo padrão é encontrado em outras escolas latino-americanas. Essa interpretação não considera uma mudança presente ou futura na direção do desenvolvimento da teoria das representações sociais em termos globais, mas tampouco sugere incorporação passiva. Não obstante, uma análise aprofundada da pesquisa e teoria conduzida em contextos variados é necessária para atingir um diagnóstico mais confiável.

O segundo fator marca a peculiaridade dos estudos relacionados à educação, geralmente publicados com amostras de professores e estudantes, contrastando com pesquisa sociopsicológica mais geral e estudos de saúde e suas associações correspondentes de grupos e objetos. O segun- do fator não se associa a continentes específicos, referindo-se a oposições amplas no campo.

Em síntese, os resultados são de certo modo uma atualização de pesquisas anteriores conduzidas no campo, e de outro modo fornecem uma cobertura mais refinada e específica da pesquisa em representações sociais, na medida em que somente periódicos foram incluídos. De Rosa e D'Ambrosio (2008) ressaltaram um contraste entre pesquisas sediadas na Europa mais relacionadas a questões teóricas e claramente alinhadas com formas paradigmáticas da teoria das representações sociais com resumos de conferências empíricos e aplicados escritos por autores latino-americanos que fizeram referência às representações sociais genericamente. Ainda que nosso estudo não tenha identificado paradigmas ou dado atenção especial aos aspectos teóricos tratados e também tenha restringido seu escopo majoritariamente a artigos empíricos, uma oposição nítida foi encontrada na análise de correspondências sugerindo uma associação mais forte entre estudos publicados em periódicos de psicologia envolvendo grupos e objetos culturais e territoriais, o que provavelmente indica uma proporção mais forte de pesquisas de base ligando com propriedades teóricas das representações sociais que os estudos sediados na América do Sul com grupos e objetos relacionados à saúde. Deve ser notado, contudo, que objetos relacionados à esfera da saúde são dominantes nos estudos empíricos da maior parte dos continentes e países, mesmo se parecem sê-lo mais na América do Sul, Brasil e Colômbia. A maior parte dos resultados de Madiot (2013) relacionados às pesquisas das Conferências Internacionais sobre Representações Sociais (CIRS) também se refletem na produção em periódicos, com forte importância de objetos de trabalho, educação e saúde e profissionais de saúde e educação como grupos de interesse, um padrão que é encontrado em toda parte nas análises apresentadas aqui, mesmo com categorias ligeiramente diferentes.

Grande parte dos resultados fornecidos por De Rosa (2013) não podem ser comparados com os apresentados neste artigo. De Rosa lidou majoritariamente com uma avaliação dos enquadramentos teóricos das representações sociais em 
publicações. Seus sistemas de categorias também são um tanto diferentes quanto aos temas de interesse nas pesquisas analisadas. Mesmo assim, alguns resultados importantes encontrados pela autora tem um paralelo em nossa pesquisa: a área temática de saúde-doença envolve uma proporção importante de $15 \%$, a maior delineando uma classe específica de objetos. A proporção global encontrada em nosso estudo $(31,1 \%)$ inclui somente os resumos com objetos sociais identificáveis; se os resumos sem essa informação fossem incluídos, a proporção seria de $20,6 \%$, que é próxima da de De Rosa, mesmo enfatizando a diferenças em termos de tipos de fontes de dados cobertos por cada estudo e sistemas de categorias, bem como uma não-coincidência quanto à noção de áreas temáticas e objetos sociais. Outro padrão dos resultados de De Rosa que foi encontrado no nosso estudo foi a associação envolvendo estudos latino-americanos e áreas temáticas aplicadas.

É importante apontar que uma parte considerável das contribuições desenvolvidas de alto nível relacionadas à pesquisa em representações sociais não foi considerada por nossa análise. Ainda que o escopo do estudo tenha envolvido basicamente artigos com grupos e objetos identificáveis, é claro que todas as contribuições apresentadas na forma de capítulos de livros, empíricos ou teóricos, não foram consideradas. Quantas são? O banco de dados de De Rosa (2013) de 2056 fontes bibliográficas tinha os capítulos de livros como a mais frequente, englobando $46,2 \%$ de todas as fontes; os artigos de periódicos foram responsáveis por $45,3 \%$. Além disso, o presente estudo incluiu algumas bases de dados importantes, mas elas foram restritas em número. Então o leitor não deveria ter o entendimento de que a análise presente cobre tudo relevante já publicado relacionado às representações sociais; em vez disso, sua meta foi de fornecer um panorama completo de artigos relevantes publicados em algumas bases de dados. Não podemos afirmar que os padrões encontrados seriam (ou não seriam) reproduzidos em todo o universo de artigos científicos e outros trabalhos. Reconhecemos que publicações importantes po- dem não ter sido recuperadas por nosso procedimento, mas ao mesmo tempo estamos confiantes de que uma parte significativa da produção publicada e indexada relacionada às representações sociais pôde ser obtida. Com algumas sobre ou sub-representações justificadas pelas bases de dados escolhidas, a imagem que emerge dos resultados é provavelmente fiel à realidade da pesquisa em representações sociais. É evidente que a produção de anais de conferências e livros tem características diferentes e sua inclusão ou consideração em separado provavelmente indicaria padrões diferentes. Por exemplo, a produção em conferências poderia incluir pesquisas mais práticas e aplicadas sem inovação suficiente para ser publicada num periódico; por outro lado, livros poderiam ser veículos privilegiados para a discussão teórica. Não obstante, a análise da produção em periódicos é provavelmente o melhor curso de ação para avaliar o nível mais alto de produção acadêmica num campo, devido ao processo de revisão por pares que é realizado, que infelizmente não é sempre o caso em conferências ou livros.

Todavia, a despeito da precisão do panorama das publicações em periódicos relacionadas às representações sociais apresentado aqui e o conhecimento que vai junto - preferências e prioridades de disciplinas, continentes e períodos, associações de grupos e objetos -, uma questão que não podemos discutir adequadamente com nossos dados, mas que é igualmente importante, senão mais, envolve o progresso teórico e o futuro do campo. Uma avaliação detalhada e crítica do que a teoria das representações sociais atingiu e o que pode ainda conquistar no entendimento do conhecimento e da sociedade é uma necessidade absoluta. Essa tarefa não pode ser mais adiada, mas não pode avançar aqui. Em termos da relevância do presente estudo, é nossa posição que o sucesso ou pertinência de uma teoria ou campo de estudo é certamente refletido em sua difusão e práticas de publicação, e essa contribuição apresenta um panorama de uma parte da produção relacionada. Esperamos que possa ajudar a comunidade científica interessada pelas representações sociais a entender o que 
foi feito até o momento, e servir como material de reflexão em discussões tentando explicar as características do campo e suas consequências.

\section{Referências}

Abric, J.-C. (Ed.). (2003). Méthodes d'étude des représentations sociales. Toulouse, France: ERES.

Alonso-Gamboa, J. O., \& Russell, J. (2012). Latin American scholarly databases: A look back to the way forward. Aslib Proceedings, 64, 32-45. doi:10.1108/00012531211196693

Arruda, A. (1987). A Psicologia Social no nordeste nos anos 80: Levantamento de dados. Revista de Psicologia, 5, 73-85.

Arruda, A. (2005). Pesquisa em representações sociais: A produção em 2003. In M. S. S. Menin \& A. M. Shimizu (Eds.), Experiência e representação social (pp. 59-92). São Paulo, SP: Casa do Psicólogo.

Camargo, B. V., Wachelke, J. F. R., \& Aguiar, A. (2007). Um panorama do desenvolvimento metodológico das pesquisas sobre representações sociais através de grupos de trabalho das jornadas internacionais. In A. S. P. Moreira \& B. V. Camargo (Eds.), Contribuições para a teoria $e$ o método de estudo das representações sociais (pp. 181-202). João Pessoa, PB: Editora da Universidade Federal da Paraíba.

Collazo-Reyes, F. (2014). Growth of the number of indexed journals of Latin America and the Caribbean: The effect on the impact of each country. Scientometrics, 98, 197-209. doi:10.1007/ s11192-013-1036-2

De Rosa, A. S. (1992). Thematic perspectives and epistemic principles in developmental social cognition and social representation: The meaning of a developmental approach to the investigation of social representation. In M. von Cranach, W. Doise, \& G. Mugny (Eds.), Social representations and the social bases of knowledge (pp. 120-143). Lewiston, NY: Hogrefe and Huber.

De Rosa, A. S. (1993). Social representations and attitudes: Problems of coherence between the theoretical definition and procedure of research. Papers on Social Representations, 2, 178-192.

De Rosa, A. S. (2013). Research fields in social representations: Snapshot views from a meta-theoret- ical analysis. In A. S. De Rosa (Ed.), Social representations in the 'social arena' (pp. 89-124). London: Routledge.

De Rosa, A. S., \& D’Ambrosio, M. (2008). International conferences as interactive scientific media channels: The history of the social representations theory through the eight editions of ICSR from Ravello (1992) to Rome (2006) [Special issue]. Rassegna di Psicologia, 2, 161-207.

Durkheim, E. (1898). Représentations individuelles et représentations collectives. Sociologie et Philosophie. Paris: Presses Universitaires de France.

Duveen, G., \& De Rosa, A. S. (1992). Social representations and the genesis of social knowledge. Papers on Social Representations, 1, 94-108.

Farr, R. M. (1993). The theory of social representations: Whence and whither? Papers on Social Representations, 2, 130-138.

Farr, R. M. (1996). The roots of modern social psychology: 1872-1954. Malden, MA: Blackwell.

Farr, R. M. (1998). From collective to social representations: Aller et retour. Culture and Psychology, 4, 275-296. doi:10.1177/1354067X9800400301

Greenacre, M. (2006). Tying up the loose ends in simple, multiple, joint correspondence analysis. In A. Rizzi \& M. Vichi (Eds.), Compstat 2006-Proceedings in Computational Statistics (pp. 163-185). Heidelberg, Germany: PhysicaVerlag HD. doi:10.1007/978-3-7908-1709-6 13

Greenacre, M. (2007). Correspondence analysis in practice ( $2^{\text {nd }}$ ed.). Boca Raton, FL: Chapman \& Hall. doi:10.1201/9781420011234

Husson, F., Lê, S., \& Pagès, J. (2011). Exploratory multivariate analysis by example using $R$. Boca Raton, FL: CRC.

Jesuíno, J. C. (2000). A Psicologia Social europeia. In J. Vala \& M. B. Monteiro (Eds.), Psicologia Social (4. ed., pp. 49-59). Lisboa, Portugal: Fundação Calouste Gulbenkian.

Jodelet, D. (1989). Représentations sociales: un domaine en expansion. In D. Jodelet (Ed.). Les représentations sociales (pp. 31-61). Paris: Presses Universitaires de France.

Jodelet, D. (2011). Ponto de vista: Sobre o movimento das representações sociais na comunidade científica brasileira. Temas em Psicologia, 19, 19-26. 
Jodelet, D., \& Ohana, J. (1989). Bibliographie générale sur les représentations sociales. In D. Jodelet (Ed.), Les représentations sociales (pp. 9-27). Paris: Presses Universitaires de France.

Le Roux, B., \& Rouanet, H. (2010). Multiple correspondence analysis. Los Angeles, CA: Sage. doi:10.4135/9781412993906

Lebart, L., Morineau, A., \& Piron, M. (1997). Statistique exploratoire multidimensionelle. Paris: Dunod.

Madiot, B. (2013). Analysing the French abstracts from the International Conferences of Social Representations. Papers on Social Representations, 22, 10.1-10.23.

Moreira, A. S. P., Camargo, B. V., Jesuíno, J. C., \& Nóbrega, S. M. (Eds.). (2005). Perspectivas teórico-metodológicas em representações sociais. João Pessoa, PB: Editora da Universidade Federal da Paraíba.

Moscovici, S. (1976). La psychanalyse, son image et son public. Paris: Presses Universitaires de France.

Moscovici, S. (1984). The phenomenon of social representations. In R. M. Farr \& S. Moscovici (Eds.), Social representations: Explorations in social psychology (pp. 3-69). Cambridge, UK: Cambridge University Press.

Moscovici, S. (1989). Des représentations collectives aux représentations sociales: élements pour une histoire. In D. Jodelet (Ed.), Les représentations sociales (pp. 62-86). Paris: Presses Universitaires de France.

Packer, A. L., Biojone, M. R., Antonio, I., Takenaka, R. M., García, A. P., Silva, A. C., ...Delbucio, H. C. R. F. (1998). SciELO: Uma metodologia para publicação eletrônica. Ciência da Informação, 27, 109-121. doi:10.1590/S010019651998000200002
R Core Team. (2013). R: A language and environment for statistical computing. Vienna, Austria: R Foundation for Statistical Computing. Retrieved from http://www.R-project.org/

Robette, N. (2014). GDAtools: A toolbox for the analysis of categorical data in social sciences, and specifically Geometric Data Analysis. R Package version 1.0. Retrieved from http://CRAN.Rproject.org/package $=$ GDAtools

Rouquette, M.-L. (1998). La communication sociale. Paris: Dunod.

Sá, C. P., \& Arruda, A. (2000). O estudo das representações sociais no Brasil [Número especial]. Revista de Ciências Humanas, 11-31.

Silva, A. M. F., Martini, J. G., \& Becker, S. G. (2011). A teoria das representações sociais nas dissertações e teses em Enfermagem: Um perfil bibliométrico. Texto e Contexto Enfermagem, 20, 294300. doi:10.1590/S0104-07072011000200011

Silva, S. E. D., Camargo, B. V., \& Padilha, M. I. (2011). A teoria das representações sociais nas pesquisas da Enfermagem brasileira. Revista Brasileira de Enfermagem, 64, 947-951. doi:10.1590/S0034-71672011000500022

Sousa, C. P. (2002). Estudos de representações sociais em Educação. Psicologia da Educação, 14/15, 285-323.
Recebido: 25/03/2014

$1^{a}$ revisão: 11/07/2014

Aceite final: 29/07/2014 